Article

\title{
Generalized Preinvex Functions and Their Applications
}

\author{
Adem Kiliçman ${ }^{1}\left(\mathbb{D}\right.$ and Wedad Saleh ${ }^{2, *}$ \\ 1 Department of Mathematics, Putra University of Malaysia (UPM), Serdang 43400, Malaysia; \\ akilic@upm.edu.my \\ 2 Department of Mathematics, Taibah University, Al-Medina 20012, Saudi Arabia \\ * Correspondence: wed_10_777@hotmail.com
}

Received: 6 September 2018; Accepted: 11 October 2018; Published: 13 October 2018

\begin{abstract}
A class of function called sub-b-s-preinvex function is defined as a generalization of s-convex and b-preinvex functions, and some of its basic properties are presented here. The sufficient conditions of optimality for unconstrainded and inquality constrained programming are discussed under the sub-b-s-preinvexity. Moreover, some new inequalities of the Hermite-Hadamard type for differentiable sub-b-s-preinvex functions are presented. Examples of applications of these inequalities are shown.
\end{abstract}

Keywords: generalized convexity; b-vex functions; sub-b-s-convex functions

\section{Introduction}

Convex functions play an important role in economics, management science, engineering, finance, and optimization theory. Many interesting generalizations and extensions of classical convexity have been used in optimization and mathematical inequalities. Generalized convex functions called b-vex functions were introduced by Bector and Singh [1], and some of their basic properties have been discussed. Choa et al. [2] investigated a new class of functions called sub-b-convex functions and proved the sufficient conditions of optimality for both unconstrained and inequality-constrained sub-b-convex programming. Hudzik and Maligranda [3] studied certain classes of functions introduced by Orlicz [4], namely, the classes of s-convex functions. Meftah [5] introduced a new class of non-negative functions called s-preinvex functions in the second sense with respect to $\eta$, for some $s \in(0,1]$. Jiagen and Tingsong Du [6] presented a class of generalized convex function has some similar properties of sub-b-convex function and s-convex functions.

Ben-Isreal and Mond [7] defined preinvex functions, and, in [8], Weir and Mond studied how and where preinvex functions could replace convex functions. Mohan and Neogy [9] presented certain properties of preinvex functions. Suneja et al. [10] considered a class of function called b-preinvex functions that are generalizations of preinvex and b-vex functions. A generalization of the b-vex function, called semi-b-preinvex, was given by Long et al. [11]. Refinements of the mathematical inequalities on convex and generalized convex functions have been investigated [12-20].

Motivated by earlier research works [6,12,21-23], the purpose of this article is to present a new class of functions, called sub-b-s-preinvex functions, that can be reduced to sub-b-preinvex when $s=1$. Some of their properties are studied. Furthermore, a new class of sets, called sub-b-s-preinvex sets, is defined. A new sub-b-s-preinvex programming is introduced, and the sufficient conditions of optimality under this type of function is established. Moreover, some examples of applications are given. 


\section{Preliminaries}

Throughout the paper, the convention bellow will be followed:

Let $\mathbb{R}^{n}$ denote the $\mathrm{n}$-dimensional Euclidean space, and let $K$ be a non-empty convex subset in $\mathbb{R}^{n}$. In addition, let $b\left(u_{1}, u_{2}, t\right): K \times K \times[0,1] \longrightarrow \mathbb{R}$ and $\eta: K \times K \longrightarrow \mathbb{R}^{n}$ be two fixed mappings.

The following definitions about b-vex, sub-b-convex, s-convex, sub-b-s-convex, and preinvex functions that will be used throughout the paper are given:

Definition 1 ([1]). The function $h: K \longrightarrow \mathbb{R}^{n}$ is called

1. $a b$-vex function on $K$ with respect to (w.r.t. in short) $b$ if

$$
h\left(t u_{1}+(1-t) u_{2}\right) \leq t b h\left(u_{1}\right)+(1-t b) h\left(u_{2}\right), \forall u_{1}, u_{1} \in K, t \in[0,1]
$$

2. and $a$ b-linear function on $K$ w.r.t. $b$ if

$$
h\left(t u_{1}+(1-t) u_{2}\right)=t b h\left(u_{1}\right)+(1-t b) h\left(u_{2}\right), \forall u_{1}, u_{1} \in K, t \in[0,1] .
$$

Definition 2 ([2]). The function $h: K \longrightarrow \mathbb{R}^{n}$ is called a sub-b-convex function on $K$ w.r.t. $b$ if

$$
h\left(t u_{1}+(1-t) u_{2}\right)=\operatorname{th}\left(u_{1}\right)+(1-t) h\left(u_{2}\right)+b\left(u_{1}, u_{2}, t\right), \forall u_{1}, u_{1} \in K, t \in[0,1] .
$$

Definition 3 ([3]). The function $h: K \longrightarrow \mathbb{R}^{n}$ is called an s-convex function in the second sense if

$$
h\left(t u_{1}+(1-t) u_{2}\right)=t^{s} h\left(u_{1}\right)+(1-t)^{s} h\left(u_{2}\right), \forall u_{1}, u_{1} \in K, t \in[0,1], s \in(0,1] .
$$

Definition 4 ([6]). A function $h: K \longrightarrow \mathbb{R}$ is called a sub-b-s-convex function on a non-empty convex set $K \subset \mathbb{R}^{n}$ w.r.t. b: $K \times K \times[0,1] \longrightarrow \mathbb{R}$ if

$$
h\left(t u_{1}+(1-\delta) u_{2}\right) \leq t^{s} h\left(u_{1}\right)+(1-t)^{s} h\left(u_{2}\right)+b\left(u_{1}, u_{2}, t\right)
$$

and $\forall u_{1}, u_{2} \in K, t \in[0,1], s \in(0,1]$.

Recall [9] that, by definition, a set $K \subset \mathbb{R}^{n}$ is called an invex set w.r.t $\eta$ if $u_{2}+t \eta\left(u_{1}, u_{2}\right) \in K$, $\forall u_{1}, u_{2} \in K$ and $t \in[0,1]$.

Ben-Israel and Mond [7] defined a class of functions called preinvex in the non-empty invex set $K \subset \mathbb{R}^{n}$ w.r.t. $\eta$, as follows:

Definition 5. A function $h: K \longrightarrow \mathbb{R}$ is preinvex on $K$ w.r.t. $\eta$ if there exists an $n$-dimensional vector function $\eta$ such that

$$
h\left(u_{2}+\delta \eta\left(u_{1}, u_{2}\right)\right) \leq \operatorname{th}\left(u_{1}\right)+(1-t) h\left(u_{2}\right)
$$

$\forall u_{1}, u_{2} \in K, t \in[0,1]$.

\section{Sub-b-s-Preinvex Function and Their Properties}

In this section, the concepts of sub-b-s-preinvex function and sub-b-s-preinvex set are given. Furthermore, some of their properties are studied.

Definition 6. A function $h: K \longrightarrow \mathbb{R}$ is called a sub-b-s-preinvex function on a non-empty invex set $K \subset \mathbb{R}^{n}$ w.r.t. $\eta, b$ if

$$
h\left(u_{2}+t \eta\left(u_{1}, u_{2}\right)\right) \leq t^{s} h\left(u_{1}\right)+(1-t)^{s} h\left(u_{2}\right)+b\left(u_{1}, u_{2}, t\right)
$$

where $b: K \times K \times[0,1] \longrightarrow \mathbb{R}, \forall u_{1}, u_{2} \in K t \in[0,1], s \in(0,1]$. 


\section{Remark 7.}

1. If $\eta\left(u_{1}, u_{2}\right)=u_{1}-u_{2}$ in Equation (1), then sub-b-s-preinvex w.r.t. $\eta, b$ becomes a sub-b-s-convex function. Moreover, if $s=1$, then Equation (1) becomes a sub-b-convex function.

2. When $\eta\left(u_{1}, u_{2}\right)=u_{1}-u_{2}$ and $b(u, u, t) \leq 0$ in Equation (1), then the sub-b-s-preinvex function becomes a convex function.

Theorem 8. If $h_{1}, h_{2}: K \longrightarrow \mathbb{R}$ are sub-b-s-preinvex functions w.r.t. $\eta, b$, then $h_{1}+h_{2}$ and $\beta h_{1}(\beta \geq 0)$ are also sub-b-s-preinvex functions w.r.t. $\eta, b$.

Corollary 9. If $h_{k}: K \longrightarrow \mathbb{R}$, where $k=1,2, \cdots, n$ are sub-b-s-preinvex functions w.r.t. $\eta, b_{k}$, then the function which is $H=\sum_{k=1}^{n} a_{k} h_{k}, a_{k} \geq 0(k=1,2, \cdots, n)$ is also sub-b-s-preinvex function w.r.t. $\eta$, $b$ where $b=\sum_{k=1}^{n} a_{k} b_{k}$.

Proposition 10. If $h_{k}: K \longrightarrow \mathbb{R}$, where $k=1,2, \cdots, n$ are sub-b-s-preinvex functions w.r.t. $\eta, b_{k}$, then the function which is $H=\max h_{k}, k=1,2, \cdots, n$ is also a sub-b-s-preinvex function w.r.t. $\eta, b$, where $b=\max b_{k}$.

Theorem 11. Let $h_{1}: K \longrightarrow \mathbb{R}$ be a sub-b-s-preinvex function w.r.t. $\eta, b_{1}$ and $h_{2}: \mathbb{R} \longrightarrow \mathbb{R}$ be an increasing function. Then $h_{1} o h_{2}$ is a sub-b-s-preinvex function w.r.t. $\eta, b$ where $b=h_{2} o b_{1}$ if $h_{2}$ satisfies the following conditions:

1. $h_{2}\left(\beta u_{1}\right)=\beta h_{2}\left(u_{1}\right), \forall u_{1} \in \mathbb{R}, \beta \geq 0$;

2. $h_{2}\left(u_{1}+u_{2}\right)=h_{2}\left(u_{1}\right)+h_{2}\left(u_{2}\right),, \forall u_{1}, u_{2} \in \mathbb{R}, \beta \geq 0$.

Proof.

$$
\begin{aligned}
\left(h_{2} o h_{1}\right)\left(u_{2}+\delta \eta\left(u_{1}, u_{2}\right)\right) & =h_{2}\left(h_{1}\left(u_{2}+t \eta\left(u_{1}, u_{2}\right)\right)\right) \\
& \leq h_{2}\left(t^{s} h_{1}\left(u_{1}\right)+(1-t)^{s} h_{1}\left(u_{2}\right)+b_{1}\left(u_{1}, u_{2}, t\right)\right) \\
& =t^{s} h_{2}\left(h_{1}\left(u_{1}\right)\right)+(1-t)^{s} h_{2}\left(h_{1}\left(u_{2}\right)\right)+h_{2}\left(b_{1}\left(u_{1}, u_{2}, t\right)\right) \\
& =t^{s}\left(h_{2} o h_{1}\right)\left(u_{1}\right)+(1-t)^{s}\left(h_{2} o h_{1}\right)\left(u_{2}\right)+b\left(u_{1}, u_{2}, t\right),
\end{aligned}
$$

which means that $h_{2} o h_{1}$ is a sub-b-s-preinvex function w.r.t. $\eta, b$.

We introduce a definition of a sub-b-s-preinvex set w.r.t. $\eta, b$ as follows.

Definition 12. A set $K \subseteq \mathbb{R}^{n+1}$ is called a sub-b-s-preinvex set w.r.t. $\eta, b$ if

$$
\left(u_{2}+t \eta\left(u_{1}, u_{2}\right), t^{s} \beta_{1}+(1-t)^{s} \beta_{2}+b\left(u_{1}, u_{2}, t\right)\right) \in K .
$$

$\forall\left(u_{1}, \beta_{1}\right),\left(u_{2}, \beta_{2}\right) \in K, u_{1}, u_{2} \in \mathbb{R}^{n}, t \in[0,1], s \in(0,1]$ and $b: \mathbb{R}^{n} \times \mathbb{R}^{n} \times[0,1] \longrightarrow \mathbb{R}$.

The epigraph of the sub-b-s-preinvex function $h: k \longrightarrow \mathbb{R}$ can be given as

$$
G(h)=\{(u, \beta): u \in K, \beta \in \mathbb{R}, h(u) \leq \beta\} .
$$

Now, we are going to investigate characterizations of the sub-b-s-preinvex function in terms of their epigraph $G(h)$, and we start with sufficient and necessary conditions for $h$ to be a sub-b-s-preinvex function w.r.t. $\eta, b$.

Theorem 13. $h: k \longrightarrow \mathbb{R}$ is a sub-b-s-preinvex function w.r.t. $\eta, b$ iff its epigraph is also a sub-b-s-preinvex set w.r.t., , b. 
Proof. Let $h$ be a sub-b-s-preinvex and let $\left(u_{1}, \beta_{1}\right),\left(u_{2}, \beta_{2}\right) \in G(h)$. Then, by using the hypothesis, we have $h\left(u_{1}\right) \leq \beta_{1}$ and $h\left(u_{2}\right) \leq \beta_{2}$.

Moreover,

$$
\begin{aligned}
h\left(u_{2}+t \eta\left(u_{1}, u_{2}\right)\right) & \leq t^{s} h\left(u_{1}\right)+(1-t)^{s} h\left(u_{2}\right)+b\left(u_{1}, u_{2}, t\right) \\
& \leq t^{s} \beta_{1}+(1-t)^{s} \beta_{2}+b\left(u_{1}, u_{2}, t\right) .
\end{aligned}
$$

Hence,

$$
\left(u_{2}+t \eta\left(u_{1}, u_{2}\right), \beta_{1}+(1-t)^{s} \beta_{2}+b\left(u_{1}, u_{2}, t\right)\right) \in G(h) .
$$

Therefore, $G(h)$ is sub-b-s-preinvex set w.r.t. $\eta, b$.

Now, assume that $G(h)$ is a sub-b-s-preinvex set w.r.t. $\eta, b$. Then

$$
\left(u_{1}, h\left(u_{1}\right)\right),\left(u_{2}, h\left(u_{2}\right)\right) \in G(h)
$$

where $u_{1}, u_{2} \in K$.

$$
\begin{gathered}
\left(u_{2}+t \eta\left(u_{1}, u_{2}\right), t^{s} h\left(u_{1}\right)+(1-t)^{s} h\left(u_{2}\right)+b\left(u_{1}, u_{2}, \delta\right)\right) \in G(h), \text { which means that } \\
h\left(u_{2}+t \eta\left(u_{1}, u_{2}\right)\right) \leq t^{s} h\left(u_{1}\right)+(1-t)^{s} h\left(u_{2}\right)+b\left(u_{1}, u_{2}, t\right) .
\end{gathered}
$$

Then $h$ is sub-b-s-preinvex function w.r.t. $\eta, b$.

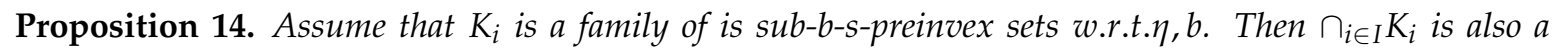
sub-b-s-preinvex set w.r.t. $\eta, b$.

Proof. Consider $\left(u_{1}, \beta_{1}\right),\left(u_{2}, \beta_{2}\right) \in \cap_{i \in I} K_{i}$. Then we have $\left(u_{1}, \beta_{1}\right),\left(u_{2}, \beta_{2}\right) \in K_{i}, \forall i \in I$

$$
\left(u_{2}+t \eta\left(u_{1}, u_{2}\right), \beta_{1}+(1-t)^{s} \beta_{2}+b\left(u_{1}, u_{2}, t\right)\right) \in K_{i}, \forall i \in I .
$$

$\Rightarrow$

$$
\left(u_{2}+t \eta\left(u_{1}, u_{2}\right), \beta_{1}+(1-t)^{s} \beta_{2}+b\left(u_{1}, u_{2}, t\right)\right) \in \cap_{i \in I} K_{i} .
$$

Hence, $\cap_{i \in I} K_{i}$ is a sub-b-s-preinvex set.

According to Theorem 13 and Proposition 14, the following proposition holds:

Proposition 15. Let $h_{i}$ be a sub-b-s-preinvex function w.r.t. $\eta$, b. Then a function $H=\sup _{i \in I} h_{i}$ is also a sub-b-s-preinvex function w.r.t. $\eta, b$.

Theorem 16. Let $h: k \longrightarrow \mathbb{R}$ be a non-negative differentiable sub-b-s-preinvex function w.r.t. $\eta, b$. Then

1. $d h_{u_{2}} \eta\left(u_{1}, u_{2}\right) \leq t^{s-1}\left(h\left(u_{1}\right)+h\left(u_{2}\right)\right)+\lim _{t \rightarrow 0_{+}} \frac{b\left(u_{1}, u_{2}, t\right)}{t}$;

2. $d h_{u_{2}} \eta\left(u_{1}, u_{2}\right) \leq t^{s-1}\left(h\left(u_{1}\right)-h\left(u_{2}\right)\right)+\frac{h\left(u_{2}\right)}{t}+\lim _{t \rightarrow 0_{+}} \frac{b\left(u_{1}, u_{2}, t\right)}{t}$.

Proof.

1. By using the hypothesis, we can write

$$
h\left(u_{2}+t \eta\left(u_{1}, u_{2}\right)\right)=h\left(u_{2}\right)+t d h_{u_{2}} \eta\left(u_{1}, u_{2}\right)+O(t) .
$$

Additionally,

$$
h\left(u_{2}+t \eta\left(u_{1}, u_{2}\right)\right) \leq t^{s} h\left(u_{1}\right)+(1-t)^{s} h\left(u_{2}\right)+b\left(u_{1}, u_{2}, t\right) .
$$


Furthermore,

$$
\begin{aligned}
h\left(u_{2}+t \eta\left(u_{1}, u_{2}\right)\right) & \leq t^{s} h\left(u_{1}\right)+(1-t)^{s} h\left(u_{2}\right)+b\left(u_{1}, u_{2}, t\right) \\
& \leq t^{s} h\left(u_{1}\right)+\left(1+t^{s}\right) h\left(u_{2}\right)+b\left(u_{1}, u_{2}, t\right) .
\end{aligned}
$$

Then

$$
h\left(u_{2}\right)+t d h_{u_{2}} \eta\left(u_{1}, u_{2}\right)+O(t) \leq t^{s} h\left(u_{1}\right)+\left(1+t^{s}\right) h\left(u_{2}\right)+b\left(u_{1}, u_{2}, t\right)
$$

by taking $\lim _{\delta \rightarrow 0_{+}} \frac{b\left(u_{1}, u_{2}, t\right)}{t}$, which is the maximum of $\frac{b\left(u_{1}, u_{2}, t\right)}{t}-\frac{O(t)}{t}$. The first result is thus obtained.

2. Similarly,

$$
\begin{aligned}
h\left(u_{2}\right)+ & t d h_{u_{2}} \eta\left(u_{1}, u_{2}\right)+O(t) \\
& \leq t^{s} h\left(u_{1}\right)+\left(1+t^{s}\right) h\left(u_{2}\right)+b\left(u_{1}, u_{2}, t\right) \\
& =t^{s} h\left(u_{1}\right)+\left(1+t^{s}\right) h\left(u_{2}\right)-t^{s} h\left(u_{2}\right)+t^{s} h\left(u_{2}\right)+b\left(u_{1}, u_{2}, t\right) \\
& =t^{s}\left(h\left(u_{1}\right)-h\left(u_{2}\right)\right)+b\left(u_{1}, u_{2}, t\right)+\left((1-t)^{s}+t^{s}\right) h\left(u_{2}\right) .
\end{aligned}
$$

However, we know that $(1-t)^{s}+\delta^{s}, \forall t \in[0,1]$, and $s \in(0,1]$ and since $h$ is non-negative function; hence,

$$
h\left(u_{2}\right)+\delta h_{u_{2}} \eta\left(u_{1}, u_{2}\right)+O(t) \leq t^{s}\left(h\left(u_{1}\right)-h\left(u_{2}\right)\right)+2 h\left(u_{2}\right)+b\left(u_{1}, u_{2}, t\right) .
$$

Then, by dividing the last inequality by $t$ and taking $\lim _{t \longrightarrow 0_{+}}$, we obtain the second part of the theorem.

Theorem 17. Let $h: k \longrightarrow \mathbb{R}$ be a negative differentiable sub-b-s-preinvex function w.r.t. $\eta, b$. Then

$$
d h_{u_{2}} \eta\left(u_{1}, u_{2}\right) \leq t^{s-1}\left(h\left(u_{1}\right)-h\left(u_{2}\right)\right)+\lim _{t \rightarrow 0_{+}} \frac{b\left(u_{1}, u_{2}, t\right)}{t} .
$$

Proof. We obtain the result by using the hypotheses, since

$$
d h_{u_{2}} \eta\left(u_{1}, u_{2}\right) \leq t^{s-1}\left(h\left(u_{1}\right)-h\left(u_{2}\right)\right)+\frac{b\left(u_{1}, u_{2}, t\right)}{t}-\frac{O(t)}{t} .
$$

Then, by taking $\lim _{t \rightarrow 0_{+}} \frac{b\left(u_{1}, u_{2}, t\right)}{t}$, which is the maximum of $\frac{b\left(u_{1}, u_{2}, t\right)}{t}-\frac{O(t)}{t}$, we obtain the result.

Corollary 18. Assume that $h: k \longrightarrow \mathbb{R}$ is a differentiable sub-b-s-preinvex function w.r.t. $\eta, b$, and

1. his a non-negative function, then

$$
d\left(h_{u_{2}}-h_{u_{1}}\right) \eta\left(u_{1}, u_{2}\right) \leq \frac{h\left(u_{1}\right)+h\left(u_{2}\right)}{t}+\lim _{t \longrightarrow 0_{+}} \frac{b\left(u_{1}, u_{2}, t\right)+b\left(u_{2}, u_{1}, t\right)}{t},
$$

2. $h$ is a negative function, then

$$
d\left(h_{u_{2}}-h_{u_{1}}\right) \eta\left(u_{1}, u_{2}\right) \leq \lim _{t \rightarrow 0_{+}} \frac{b\left(u_{1}, u_{2}, t\right)-b\left(u_{2}, u_{1}, t\right)}{t} .
$$

\section{Proof.}


1. Since $h$ is a non-negative function and by using Theorem 16,

$$
d h_{u_{2}} \eta\left(u_{1}, u_{2}\right) \leq t^{s-1}\left(h\left(u_{1}\right)-h\left(u_{2}\right)\right)+\frac{h\left(u_{2}\right)}{t}+\lim _{t \longrightarrow 0_{+}} \frac{b\left(u_{1}, u_{2}, t\right)}{t} .
$$

Additionally,

$$
d h_{u_{1}} \eta\left(u_{1}, u_{2}\right) \leq t^{s-1}\left(h\left(u_{2}\right)-h\left(u_{1}\right)\right)+\frac{h\left(u_{1}\right)}{t}+\lim _{t \longrightarrow 0_{+}} \frac{b\left(u_{2}, u_{1}, t\right)}{t} .
$$

Thus,

$$
d\left(h_{u_{2}}-h_{u_{1}}\right) \eta\left(u_{1}, u_{2}\right) \leq \frac{h\left(u_{1}\right)+h\left(u_{2}\right)}{t}+\lim _{t \longrightarrow 0_{+}} \frac{b\left(u_{1}, u_{2}, t\right)+b\left(u_{2}, u_{1}, t\right)}{t}
$$

2. Since $h$ is a negative function, and according to Theorem 17, the second result can be obtained directly.

\section{Hermite-Hadamard-Type Integral Inequalities for Differentiable Sub-B-S-Preinvex Functions}

There are a great deal of inequalities related to the class of convex functions. For example, Hermite-Hadamard's inequality is one of the well-known results in the literature, which can be stated as follows.

Theorem 19. (Hermite-Hadamard's inequality) Let $h$ be a convex function on $\left[u_{1}, u_{2}\right]$ with $u_{1}<u_{2}$. If $h$ is an integral on $\left[u_{1}, u_{2}\right]$, then

$$
h\left(\frac{u_{1}+u_{2}}{2}\right) \leq \frac{1}{u_{2}-u_{1}} \int_{u_{1}}^{u_{2}} h(x) d x \leq \frac{h\left(u_{1}\right)+h\left(u_{2}\right)}{2} .
$$

For more properties about the above inequality, we refer the interested readers to [24,25]. Dragomir and Fitzpatrick [26] demonstrated a variation of Hadamard's inequality, which holds for s-convex functions in the second sense.

Theorem 20. Theorem Let $h: \mathbf{R}_{+} \longrightarrow \mathbf{R}_{+}$be an s-convex function in the second sense $s \in(0,1)$ and $u_{1}, u_{2} \in \mathbf{R}_{+}, u_{1}<u_{2}$. If $h \in L^{1}\left(\left[u_{1}, u_{2}\right]\right)$, then

$$
2^{s-1} h\left(\frac{u_{1}+u_{2}}{2}\right) \leq \frac{1}{u_{2}-u_{1}} \int_{u_{1}}^{u_{2}} h(x) d x \leq \frac{h\left(u_{1}\right)+h\left(u_{2}\right)}{s+1} .
$$

Now, we will present new inequalities of Hermite-Hadamard for functions whose derivatives in absolute value are sub-b-s-preinvex functions. Our results generalize those results presented in [27] concerning Hermite-Hadamard type inequalities for preinvex functions.

Lemma 21 ([27]). Assume that $K \subset \mathbf{R}$ is an open invex subset w.r.t and $u_{1}, u_{2} \in K$ with $u_{1}<u_{1}+\eta\left(u_{2}, u_{1}\right)$. Let $h: K \longrightarrow \mathbf{R}$ be a differentiable mapping on $K$ such that $h^{\prime} \in L\left(\left[u_{1}, u_{1}+\eta\left(u_{2}, u_{1}\right)\right]\right)$. Then the following equality holds:

$$
\begin{aligned}
-\frac{h\left(u_{1}\right)+h\left(u_{1}+\eta\left(u_{2}, u_{1}\right)\right)}{2}+ & \frac{1}{\eta\left(u_{2}, u_{1}\right)} \int_{u_{1}}^{u_{1}+\eta\left(u_{2}, u_{1}\right)} h(x) d x \\
& =\frac{\eta\left(u_{2}, u_{1}\right)}{2} \int_{0}^{1}(1-2 t) h^{\prime}\left(u_{1}+t \eta\left(u_{2}, u_{1}\right)\right) d t .
\end{aligned}
$$


Theorem 22. Assume that $K \subset[0, c], c>0$ is an open invex subset w.r.t $\eta$ and $u_{1}, u_{2} \in K$ with $u_{1}<u_{1}+\eta\left(u_{2}, u_{1}\right)$. Let $h: K \longrightarrow \mathbf{R}$ be a differentiable mapping on $K$ such that $h^{\prime} \in L\left(\left[u_{1}, u_{1}+\eta\left(u_{2}, u_{1}\right)\right]\right)$. If $\left|h^{\prime}\right|$ is a sub-b-s-preinvex function on $K$, then we have the following inequality:

$$
\begin{aligned}
& \left|\frac{h\left(u_{1}\right)+h\left(u_{1}+\eta\left(u_{2}, u_{1}\right)\right)}{2}-\frac{1}{\eta\left(u_{2}, u_{1}\right)} \int_{u_{1}}^{u_{1}+\eta\left(u_{2}, u_{1}\right)} h(x) d x\right| \\
& \leq \frac{\eta\left(u_{2}, u_{1}\right)}{2}\left[\frac{\left(2^{s+1}-1\right)(s+1)+\left(1-2^{s}\right)(s+2)}{2^{s}(s+1)(s+2)}\left[\left|h^{\prime}\left(u_{2}\right)\right|+\left|h^{\prime}\left(u_{1}\right)\right|\right]+\frac{1}{2}\left|b\left(u_{1}, u_{2}, t\right)\right|\right]
\end{aligned}
$$

Proof. From Lemma 21, we have

$$
\begin{gathered}
\left|\frac{h\left(u_{1}\right)+h\left(u_{1}+\eta\left(u_{2}, u_{1}\right)\right)}{2}-\frac{1}{\eta\left(u_{2}, u_{1}\right)} \int_{a}^{u_{1}+\eta\left(u_{2}, u_{1}\right)} h(x) d x\right| \\
\leq \frac{\eta\left(u_{2}, u_{1}\right)}{2} \int_{0}^{1}|1-2 t|\left|h^{\prime}\left(u_{1}+t \eta\left(u_{2}, u_{1}\right)\right)\right| d t
\end{gathered}
$$

Since $\left|h^{\prime}\right|$ is a sub-b-s-preinvex on $K$, for every $u_{1}, u_{2} \in K, \in(0,1]$ and $s \in(0,1)$, we obtain

$$
\left|h^{\prime}\left(u_{1}+t \eta\left(u_{2}, u_{1}\right)\right)\right| \leq t^{s}\left|h^{\prime}\left(u_{2}\right)\right|+(1-t)^{s}\left|h^{\prime}\left(u_{1}\right)\right|+\left|b\left(u_{1}, u_{2}, t\right)\right| .
$$

Hence, we have

$$
\begin{aligned}
& \left|\frac{h\left(u_{1}\right)+h\left(u_{1}+\eta\left(u_{1}, u_{2}\right)\right)}{2}-\frac{1}{\eta\left(u_{1}, u_{2}\right)} \int_{a}^{u_{1}+\eta\left(u_{1}, u_{2}\right)} h(x) d x\right| \\
\leq & \frac{\eta\left(u_{1}, u_{2}\right)}{2}\left[\left|h^{\prime}\left(u_{2}\right)\right| \int_{0}^{1}|1-2 t| t^{s} d t+\left|h^{\prime}\left(u_{1}\right)\right| \int_{0}^{1}|1-2 t|(1-t)^{s} d t+\left|b\left(u_{1}, u_{2}, t\right)\right| \int_{0}^{1}|1-2 t| d t\right] .
\end{aligned}
$$

since

$$
\begin{aligned}
\int_{0}^{1} 1-2 t \mid(1-t)^{s} d t & =\int_{0}^{1} 1-2 t \mid t^{s} d t \\
& =\int_{0}^{\frac{1}{2}}(1-2 t) t^{s} d t-\int_{\frac{1}{2}}^{1}(1-2 t) t^{s} d t \\
& =\frac{\left(2^{s+1}-1\right)(s+1)+\left(1-2^{s}\right)(s+2)}{2^{s}(s+1)(s+2)}
\end{aligned}
$$

Additionally,

$$
\int_{0}^{1}|1-2 t| d t=\frac{1}{2}
$$

Therefore, the proof of Theorem 22 is complete.

Corollary 23. If $\eta\left(u_{2}, u_{1}\right)=u_{2}-u_{1}$ in Theorem 22, then Inequality 4 reduces to the following inequality:

$$
\begin{gathered}
\left|\frac{h\left(u_{1}\right)+h\left(u_{2}\right)}{2}-\frac{1}{u_{2}-u_{1}} \int_{u_{1}}^{u_{2}} h(x) d x\right| \\
\leq \frac{u_{2}-u_{1}}{2}\left[\frac{\left(2^{s+1}-1\right)(s+1)+\left(1-2^{s}\right)(s+2)}{2^{s}(s+1)(s+2)}\left[\left|h^{\prime}\left(u_{2}\right)\right|+\left|h^{\prime}\left(u_{1}\right)\right|\right]+\frac{1}{2}\left|b\left(u_{1}, u_{2}, t\right)\right|\right] .
\end{gathered}
$$


Theorem 24. Assume that $K \subset[0, c], c>0$ is an open invex subset w.r.t $\eta$ and $u_{1}, u_{2} \in K$ with $u_{1}<u_{1}+\eta\left(u_{2}, u_{1}\right)$. Let $h: K \longrightarrow \mathbf{R}$ be a differentiable mapping on $K$ such that $h^{\prime} \in L\left(\left[u_{1}, u_{1}+\eta\left(u_{2}, u_{1}\right)\right]\right)$. If $\left|h^{\prime}\right|^{q}$ is a sub-b-s-preinvex function on $K$ for $q>1$, then we have the following inequality:

$$
\begin{aligned}
\mid \frac{h\left(u_{1}\right)+h\left(u_{1}+\eta\left(u_{2}, u_{1}\right)\right)}{2} & -\frac{1}{\eta\left(u_{2}, u_{1}\right)} \int_{u_{1}}^{u_{1}+\eta\left(u_{2}, u_{1}\right)} h(x) d x \mid \\
\leq & \frac{\eta\left(u_{2}, u_{1}\right)}{2(p+1)^{\frac{1}{p}}}\left[\frac{\left|h^{\prime}\left(u_{2}\right)\right|^{q}+\left|h^{\prime}\left(u_{1}\right)\right|^{q}}{s+1}+\left|b\left(u_{1}, u_{2}, t\right)\right|\right]^{\frac{1}{q}}
\end{aligned}
$$

where $\frac{1}{p}+\frac{1}{q}=1$.

Proof. From Lemma 21 and using the Hölder's integral inequality, we have

$$
\begin{aligned}
& \left|\frac{h\left(u_{1}\right)+h\left(u_{1}+\eta\left(u_{2}, u_{1}\right)\right)}{2}-\frac{1}{\eta\left(u_{2}, u_{1}\right)} \int_{a}^{u_{1}+\eta\left(u_{2}, u_{1}\right)} h(x) d x\right| \\
& \leq \frac{\eta\left(u_{2}, u_{1}\right)}{2}\left(\int_{0}^{1}|1-2 t|^{p} d t\right)^{\frac{1}{p}}\left(\int_{0}^{1}\left|\hat{h}\left(u_{1}+t \eta\left(u_{2}, u_{1}\right)\right)\right|^{q} d t\right)^{\frac{1}{q}} .
\end{aligned}
$$

Since $\left|h^{\prime}\right|^{q}$ is a sub-b-s-preinvex on $K$, for every $u_{1}, u_{2} \in K, \in(0,1]$ and $s \in(0,1)$, we obtain

$$
\left|h^{\prime}\left(u_{1}+t \eta\left(u_{2}, u_{1}\right)\right)\right|^{q} \leq t^{s}\left|h^{\prime}\left(u_{2}\right)\right|^{q}+(1-t)^{s}\left|h^{\prime}\left(u_{1}\right)\right|^{q}+\left|b\left(u_{1}, u_{2}, t\right)\right| .
$$

Hence,

$$
\begin{aligned}
\int_{0}^{1}\left|h^{\prime}\left(u_{1}+t \eta\left(u_{2}, u_{1}\right)\right)\right|^{q} d t & \leq\left[\left|h^{\prime}\left(u_{2}\right)\right|^{q}+\left|h^{\prime}\left(u_{1}\right)\right|^{q}\right] \int_{0}^{1} t^{s} d t+\left|b\left(u_{1}, u_{2}, t\right)\right| \\
& =\frac{1}{s+1}\left[\left|h^{\prime}\left(u_{2}\right)\right|^{q}+\left|h^{\prime}\left(u_{1}\right)\right|^{q}\right]+\left|b\left(u_{1}, u_{2}, t\right)\right|
\end{aligned}
$$

Moreover, via basic calculus, we obtain $\int_{0}^{1}|1-2 t|^{p} d t=\frac{1}{p+1}$. Thus, the proof of Theorem 24 is complete.

Corollary 25. If $\eta\left(u_{2}, u_{1}\right)=u_{2}-u_{1}$ in Theorem 24 , then Inequality 8 reduces to the following inequality:

$$
\begin{aligned}
& \left|\frac{h\left(u_{1}\right)+h\left(u_{2}\right)}{2}-\frac{1}{u_{2}-u_{1}} \int_{u_{1}}^{u_{2}} h(x) d x\right| \\
& \leq \frac{u_{2}-u_{1}}{2(p+1)^{\frac{1}{p}}}\left[\frac{\left|\hat{h}\left(u_{2}\right)\right|^{q}+\left|\hat{h}\left(u_{1}\right)\right|^{q}}{s+1}+\left|b\left(u_{1}, u_{2}, t\right)\right|\right]^{\frac{1}{q}}
\end{aligned}
$$

where $\frac{1}{p}+\frac{1}{q}=1$.

\section{Application}

In this section, we apply our results to the non-linear programming problem and to special means. Let us consider the unconstraint problem $(P)$

$$
(P): \min \{h(u), u \in K\} \text {. }
$$


Theorem 26. Consider that $h: k \longrightarrow \mathbb{R}$ is a non-negative differentiable sub-b-s-preinvex function w.r.t. $\eta, b$. If $u^{*} \in K$ and

$$
d h_{u^{*}} \eta\left(u, u^{*}\right) \geq \frac{h\left(u^{*}\right)}{t}+\lim _{t \longrightarrow 0_{+}} \frac{b\left(u, u^{*}, t\right)}{t}, \forall u \in K, t \in[0,1], s \in(0,1],
$$

then $u^{*}$ is the optimal solution to $(P)$ with respect to $h$ on $K$.

Proof. By using the hypothesis and the second pair of Theorem 16, we obtain

$$
d h_{u^{*}} \eta\left(u, u^{*}\right)-\frac{h\left(u^{*}\right)}{t}-\lim _{t \rightarrow 0_{+}} \frac{b\left(u, u^{*}, t\right)}{t} \leq t^{s-1}\left(h(u)-h\left(u^{*}\right)\right),
$$

$\forall t \in[0,1], s \in(0,1]$, and since

$$
d h_{u^{*}} \eta\left(u, u^{*}\right) \geq \frac{h\left(u^{*}\right)}{t}+\lim _{t \rightarrow 0_{+}} \frac{b\left(u, u^{*}, t\right)}{t} .
$$

That is $h(u)-h\left(u^{*}\right) \geq 0$, which means that $u^{*}$ is the optimal solution.

Example 27. Let us take the following function $h: \mathbb{R}^{+} \longrightarrow \mathbb{R}$ such that $h(u)=2 u^{s}$, where $s \in(0,1]$. Additionally, let $b\left(u_{1}, u_{2}, t\right)=t u_{1}^{2}+4 t u_{2}^{2}$ and

$$
\eta\left(u_{1}, u_{2}\right)=\left\{\begin{array}{l}
-u_{2} ; u_{1}=u_{2} \\
1-u_{2} ; u_{1} \neq 2 .
\end{array}\right.
$$

Since $b\left(u_{1}, u_{2}, t\right) \geq 0, \forall t \in(0,1]$, it is easy to say that $h$ is a sub-b-s-preinvex function. Additionally, $h(u)$ is a non-negative differentiable, and $\lim _{t \rightarrow 0_{+}} \frac{b\left(u_{1}, u_{2}, t\right)}{t}$ exists for every $u_{1}, u_{2} \in \mathbb{R}^{+}$and $t \in(0,1]$. Thus, the following unconstraint sub-b-s-preinvex programming can be given as

$$
\begin{gathered}
(P): \min \left\{h(u), u \in \mathbb{R}^{+}\right\} . \\
d h_{u^{*}} \eta\left(u, u^{*}\right)=2 s\left(u^{*}\right)^{s-1} \eta\left(u, u^{*}\right), \frac{h\left(u^{*}\right)}{t}=\frac{2\left(u^{*}\right)^{s}}{t}
\end{gathered}
$$

and

$$
\lim _{t \rightarrow 0_{+}} \frac{b\left(u, u^{*}, t\right)}{t}=u^{2}+4\left(u^{*}\right)^{2}
$$

Thus, we see that $u^{*}=0$ and

$$
d h_{u^{*}} \eta\left(u, u^{*}\right) \geq \frac{h\left(u^{*}\right)}{t}+\lim _{t \rightarrow 0_{+}} \frac{b\left(u, u^{*}, t\right)}{t}
$$

holds $\forall u \in K, t \in(0,1], s \in(0,1)$. Hence, according to Theorem 26 , the minimum value of $h(u)$ at zero.

Corollary 28. Assume that $h: K \longrightarrow \mathbb{R}$ is a strictly non-negative differentiable sub-b-s-preinvex function w.r.t. $\eta, b$. If $u^{*} \in K$ and satisfies the condition of Equation (12), then $u^{*}$ is the unique optimal solution of $h$ on $\mathrm{K}$.

Proof. Since $h$ is a strictly non-negative differentiable sub-b-s-preinvex function w.r.t. $\eta, b$ and by using Theorem 16, we obtain

$$
d h_{u_{2}} \eta\left(u_{1}, u_{2}\right)<t^{s-1}\left(h\left(u_{1}\right)-h\left(u_{2}\right)\right)+\frac{h\left(u_{2}\right)}{t}+\lim _{t \longrightarrow 0_{+}} \frac{b\left(u_{1}, u_{2}, t\right)}{t} .
$$


Let $v_{1}, v_{2} \in K$ where $v_{1} \neq v_{2}$ be optimal solutions of $(P)$. Then $h\left(v_{1}\right)=h\left(v_{2}\right)$, and Equation (13) yields that

$$
d h_{v_{2}} \eta\left(v_{1}, v_{2}\right)-\frac{h\left(v_{2}\right)}{t}-\lim _{t \longrightarrow 0_{+}} \frac{b\left(v_{1}, v_{2}, t\right)}{t}<t^{s-1}\left(h\left(v_{1}\right)-h\left(v_{2}\right)\right) .
$$

By using Equation (12), we have $t^{s-1}\left(h\left(v_{1}\right)-h\left(v_{2}\right)\right)>0$, but $h\left(v_{1}\right)=h\left(v_{2}\right)$. Thus, $v_{1}=v_{2}=u^{*}$. It follows that $u^{*}$ is the unique optimal optimal solution of $h$ on $K$.

Now, grant nonlinear programming as follows:

$$
\left(P_{*}\right): \min \left\{h(u): u \in \mathbb{R}^{n}, f_{i}(u) \leq 0, i \in I \text {, where } I=1,2, \cdots, m\right\} .
$$

$F_{e}$ is the feasible set of $\left(P_{*}\right)$, which is given as

$$
S_{f}=\left\{u \in \mathbb{R}^{n}: f_{i}(u) \leq 0, i \in I\right\} .
$$

In addition, for $u^{*} \in S_{f}$, we define $N\left(u^{*}\right)=\left\{i: f_{i}\left(u^{*}\right)=0, i \in I\right\}$.

Theorem 29 (Karush-Kuhn-Tucker Sufficient Conditions). Assume that $h: \mathbb{R}^{n} \longrightarrow \mathbb{R}$ is a non-negative differentiable sub-b-s-preinvex function w.r.t. $\eta, b$ and $f_{i}: \mathbb{R}^{n} \longrightarrow \mathbb{R}$ are differentiable sub-b-s-preinvex functions w.r.t. $\eta, b_{i}, i \in I$. Additionally, let

$$
d h_{u^{*}} \eta\left(u, u^{*}\right)+\sum_{i \in I} v_{i} d f_{i u^{*}} \eta\left(u, u^{*}\right)=0, u^{*} \in S_{f}, v_{i} \geq 0, i \in I .
$$

If

$$
\frac{h\left(u^{*}\right)}{t}+\lim _{t \longrightarrow 0_{+}} \frac{b\left(u, u^{*}, t\right)}{t} \leq-\sum_{i=1} v_{i} \lim _{t \rightarrow 0_{+}} \frac{b\left(u, u^{*}, t\right)}{t},
$$

then $u^{*}$ is an optimal solution of $\left(P_{*}\right)$.

Proof. For any $u \in S_{f}$, then we obtain $f_{i}(u) \leq 0=f_{i}\left(u^{*}\right), \forall u \in S_{f}$. Therefore, from the sub-b-s-preinvexity of $f_{i}$ and Theorem 17 , we get

$$
d f_{i u^{*}} \eta\left(u, u^{*}\right)-\lim _{t \longrightarrow 0_{+}} \frac{b\left(u, u^{*}, t\right)}{t} \leq t^{s-1}\left(f_{i}(u)-f_{i}\left(u^{*}\right)\right) \leq 0 .
$$

From Equation (14), we obtain

$$
\begin{aligned}
d h_{u^{*}} \eta\left(u, u^{*}\right) & =-\sum_{i \in I} v_{i} d f_{i u^{*}} \eta\left(u, u^{*}\right) \\
& =-\sum_{i \in N\left(u^{*}\right)} v_{i} d f_{i u^{*}} \eta\left(u, u^{*}\right)
\end{aligned}
$$

Equations (15) and (17) yields that

$$
\begin{aligned}
d h_{u^{*}} \eta\left(u, u^{*}\right) & -\frac{h\left(u^{*}\right)}{t}-\lim _{t \rightarrow 0_{+}} \frac{b\left(u, u^{*}, t\right)}{t} \\
& \geq-\sum_{i \in N\left(u^{*}\right)} v_{i}\left(d f_{i u^{*}} \eta\left(u, u^{*}\right)-\lim _{t \rightarrow 0_{+}} \frac{b\left(u, u^{*}, t\right)}{t}\right) .
\end{aligned}
$$

Here, we use Equations (16) and (18) to obtain

$$
d h_{u^{*}} \eta\left(u, u^{*}\right) \geq \frac{h\left(u^{*}\right)}{t}+\lim _{t \longrightarrow 0_{+}} \frac{b\left(u, u^{*}, t\right)}{t}
$$


and according to Theorem 26, one has

$$
h(u) \geq h\left(u^{*}\right), \forall u \in S_{f} .
$$

Hence, $u^{*}$ is an optimal solution of $\left(P_{*}\right)$.

Now, some applications to special means are given. The following result is established in [28].

Assume that $H: I_{1} \longrightarrow I_{2} \subset[0, \infty)$ is a non-negative convex function on $I_{1}$. Then $H^{s}(x)$ is s-convex on $I_{1}$, where $s \in(0,1)$. For arbitrary positive real numbers $u_{1}, u_{2}\left(u_{1} \neq u_{2}\right)$, the following special means are given:

1. The arithmetic mean:

$$
A=A\left(u_{1}, u_{2}\right)=\frac{u_{1}+u_{2}}{2}, u_{1}, u_{2} \geq 0
$$

2. The logarithmic mean:

$$
L=L\left(u_{1}, u_{2}\right)=\left\{\begin{array}{ll}
u_{1} & \text { if } u_{1}=u_{2} \\
\frac{u_{2}-u_{1}}{\ln u_{1}, \ln u_{2}} & \text { if } u_{1} \neq u_{2},
\end{array} . u_{1}, u_{2}>0\right.
$$

3. The P-logarithmic mean:

$$
L_{p}=L_{p}\left(u_{1}, u_{2}\right)=\left\{\begin{array}{ll}
u_{1} & \text { if } u_{1}=u_{2}, \\
{\left[\frac{u_{2}^{p+1}-u_{1}^{p+1}}{(p+1)\left(u_{2}-u_{1}\right)}\right]^{\frac{1}{p}}} & \text { if } u_{1} \neq u_{2},
\end{array} p \in \mathbf{R}\{-1,0\}, u_{1}, u_{2}>0\right.
$$

It is well known that $L_{p}$ is monotonic non-decreasing over $p \in \mathbf{R}$ with $L_{-1}=L$ and $L_{0}=1$. In particular, we have the following inequality

$$
L \leq A
$$

Now, some new inequalities are derived for the above means

Let $h:\left[u_{1}, u_{2}\right] \longrightarrow \mathbf{R}, 0<u_{1}<u_{2}, h(x)=x^{s}$ and $s \in(0,1]$, Then

$$
\begin{gathered}
\frac{1}{u_{2}-u_{1}} \int_{u_{1}}^{u_{2}} h(x) d x=L_{s}^{s}\left(u_{1}, u_{2}\right) \\
\frac{h\left(u_{1}\right)+h\left(u_{2}\right)}{2}=A\left(u_{1}^{s}, u_{2}^{s}\right) .
\end{gathered}
$$

1. From Corollary 23,

$$
\begin{aligned}
& \left|A\left(u_{1}^{s}, u_{2}^{s}\right)-L_{s}^{s}\left(u_{1}, u_{2}\right)\right| \\
\leq & \frac{u_{2}-u_{1}}{2}\left[\frac{\left(2^{s+1}-1\right)(s+1)+\left(1-2^{s}\right)(s+2)}{2^{s}(s+1)(s+2)}\left[\left|\hat{h}\left(u_{2}\right)\right|+\left|\hat{h}\left(u_{1}\right)\right|\right]+\frac{1}{2}\left|b\left(u_{1}, u_{2}, t\right)\right|\right] .
\end{aligned}
$$

2. From Corollary 25,

$$
\left|A\left(u_{1}^{s}, u_{2}^{s}\right)-L_{s}^{s}\left(u_{1}, u_{2}\right)\right| \leq \frac{u_{2}-u_{1}}{2(p+1)^{\frac{1}{p}}}\left[\frac{\left|\hat{h}\left(u_{2}\right)\right|^{q}+\left|\hat{h}\left(u_{1}\right)\right|^{q}}{s+1}+\left|b\left(u_{1}, u_{2}, t\right)\right|\right]^{\frac{1}{q}}
$$

If $s=1$, then 


$$
\begin{gathered}
\left|A\left(u_{1}, u_{2}\right)-L\left(u_{1}, u_{2}\right)\right| \leq \frac{u_{2}-u_{1}}{4}\left[\frac{1}{2}\left[\left|h^{\prime}\left(u_{2}\right)\right|+\left|h^{\prime}\left(u_{1}\right)\right|\right]+\left|b\left(u_{1}, u_{2}, t\right)\right|\right] \\
\left|A\left(u_{1}, u_{2}\right)-L\left(u_{1}, u_{2}\right)\right| \leq \frac{u_{2}-u_{1}}{2(p+1)^{\frac{1}{p}}}\left[\frac{1}{2}\left[\left|h^{\prime}\left(u_{2}\right)\right|^{q}+\left|h^{\prime}\left(u_{1}\right)\right|^{q}\right]+\left|b\left(u_{1}, u_{2}, t\right)\right|\right]
\end{gathered}
$$

where $q>1$ and $\frac{1}{p}+\frac{1}{q}=1$.

\section{Conclusions}

In this paper, we introduce a new class of functions and sets called sub-b-s-preinvex functions and sub-b-s-preinvex sets and discuss some of their properties. In addition, the optimality conditions for a non-linear programming problem are also established. Hermite-Hadamard-type integral inequalities for differentiable sub-b-s-preinvex functions have been studied. Relationships between these inequalities and the classical inequalities have been established. The ideas and techniques of this paper may motivate further research, for example, in manifolds.

Author Contributions: Both authors have contributed equally to this paper. The idea of this whole paper was brought about by W.S., she also prepared the whole article; A.K. looked into the final manuscript.

Funding: This research received no external funding.

Conflicts of Interest: The authors declare no conflict of interest.

\section{References}

1. Bector, C.R.; Singh, C. B-vex functions. J. Optim. Theory Appl. 1991, 71, 439-453. [CrossRef]

2. Chao, M.T.; Jian, J.B.; Liang, D.Y. Sub-b-convex functions and sub-b-convex programming. Oper. Res. Trans. 2012, 16, 1-8.

3. Hudzik, H.; Maligranda, L. Some remarks on s-convex functions. Aequ. Math. 1994, 48, 100-111. [CrossRef]

4. Orlicz, W. A note on modular spaces I. Bull. Acad. Polon. Sci. Ser. Math. Astronom. Phys. 1961, 9, $157-162$.

5. Meftah, B. New integral inequalities for s-preinvex functions. Int. J. Nonlinear Anal. 2017, 8, 331-336.

6. Liao, J.; Tingsong, D. On Some Characterizations of Sub-b-s-Convex Functions. Filomat 2016, 30, $3885-3895$. [CrossRef]

7. Ben-Israel, A.; Mond, B. What is invexity? ANZIAM J. 1986, 28, 1-9. [CrossRef]

8. Weir, T.; Mond, B. Pre-invex functions in multiple objective optimization. J. Math. Anal. Appl. 1988, 136, 29-38. [CrossRef]

9. Mohan, S.R.; Neogy, S.K. On invex sets and preinvex functions. J. Math. Anal. Appl. 1995, 189, 901-908. [CrossRef]

10. Suneja, S.K.; Singh, C.; Bector, C.R. Generalization of preinvex and B-vex functions. J. Optim. Theory Appl. 1993, 76, 577-587. [CrossRef]

11. Long,X.J.; Peng, J.W. Semi-B-preinvex functions. J. Optim. Theory Appl. 2006, 131, 301-305. [CrossRef]

12. Ahmad, I.; Jayswalb, A.; Kumarib, B. Characterizations of geodesic sub-b-s-convex functions on Riemannian manifolds. J. Nonlinear Sci. Appl. 2018, 11, 189-197. [CrossRef]

13. Boltyanski, V.; Martini, H.; Soltan, P.S. Excursions into Combinatorial Geometry; Springer Science \& Business Media: Berlin/Heidelberg, Germany, 1997.

14. Chen, X. Some properties of semi-E-convex functions. J. Math. Anal. Appl. 2002, 275, 251-262. [CrossRef]

15. Duca, D.I.; Lupsa, L. On the E-epigraph of an E-convex functions. J. Optim. Theory Appl. 2006, 129, $341-348$. [CrossRef]

16. Dwilewicz, R.J. A short History of Convexity. Differ. Geom. Dyn. Syst. 2009, 11, 112-129.

17. Kiliçman, A.; Saleh, W. A note on starshaped sets in2-dimensional manifolds without conjugate points. J. Funct. Spaces 2014, 2014, 3.

18. Liu, W. New integral inequalities involving beta function via P-convexity. Miskolc Math. Notes 2014, 15, 585-591. 
19. Martini, H.; Swanepoel, K.J. Generalized convexity notions and combinatorial geometry. Gongr. Numer. 2003, 164, 65-93.

20. Syau, Y.R.; Jia, L.; Lee, E.S. Generalizations of E-convex and B-vex functions. Comput. Math. Appl. 2009, 58, 711-716. [CrossRef]

21. Liao, J.; Tingsong, D. Optimality Conditions in Sub-(b, m)-Convex Programming. Univ. Politeh. Buchar. Sci. Bull.-Ser. A-Appl. Math. Phys. 2017, 79, 95-106.

22. Fok, H.; Von, S. Generalizations of some Hermite-Hadamard-type inequalities. Indian J. Pure Appl. Math. 2015, 46, 359-370 [CrossRef]

23. Noor, M.A.; Noor, K.I.; Iftikhar, S.; Safdar, F. Some Properties of Generalized Strongly Harmonic Convex Functions. Int. J. Anal. Appl. 2018, 16, 427-436.

24. Dragomir, S.S. On Hadamards inequality for convex functions on the co-ordinates in a rectangle from the plane. Taiwan. J. Math. 2001, 5, 775-788. [CrossRef]

25. Hua, J.; Xi, B.; Feng, Q. Inequalities of Hermite-Hadamard type involving an s-convex function with applications. Appl. Math. Comput. 2014, 246, 752-760. [CrossRef]

26. Dragomir, S.S.; Fitzpatrick, S. The Hadamard inequalities for s-convex functions in the second sense. Demonstr. Math. 1999, 32, 687-696. [CrossRef]

27. Barani, A.; Ghazanfari, A.G.; Dragomir, S.S. Hermite-Hadamard inequality for functions whose derivatives absolute values are preinvex. J. Inequal. Appl. 2012, 2012, 247. [CrossRef]

28. Pearce, C.E.M.; Pecaric, J. Inequalities for differentiable mappings with application to specialmeans and quadrature formulae. Appl. Math. Lett. 2000, 13, 51-55. [CrossRef]

(C) 2018 by the authors. Licensee MDPI, Basel, Switzerland. This article is an open access article distributed under the terms and conditions of the Creative Commons Attribution (CC BY) license (http:/ / creativecommons.org/licenses/by/4.0/). 